\title{
Long-term study of the Light-curve of PKS 1510-089 in GeV energies
}

\author{
Raj Prince* \\ Raman Research Institute, Bangalore, India \\ E-mail: rajprinceerri.res.in \\ Pratik Majumdar $^{1}$, Nayantara Gupta ${ }^{2}$ \\ ${ }^{1}$ Saha Institute of Nuclear Physics, Kolkata, India \\ ${ }^{2}$ Raman Research Institute, Bangalore, India
}

We have analyzed data from the FSRQ PKS 1510-089 collected over a period of eight years from August 2008 to Dec 2016 with the Fermi-LAT. We have identified several flares of this highly variable source, studied their temporal and spectral properties in detail, and compared with previous works on flares of PKS 1510-089. Five major flares and a few sub-flares or sub-structures have been identified in our study. The fastest variability time is found to be $1.30 \pm 0.18 \mathrm{hr}$, where we estimate the minimum size of the emission region to be $4.85 \times 10^{15}$ $\mathrm{cm}$. In most of the flares, the $\gamma$-ray SEDs are better fitted with a log-parabolic distribution compared to a simple power law or a power law with exponential cutoffs. This has strong physics implications regarding the nature of the high-energy $\gamma$-ray emission region.

7th Fermi Symposium 2017

15-20 October 2017

Garmisch-Partenkirchen, Germany

\footnotetext{
* Speaker.
} 


\section{Intoduction}

PKS 1510-089, located at a redshift of $0.361[8,12]$ is highly variable and has been observed in gamma ray energies upto $400 \mathrm{GeV}[3,10]$. This highly variable Flat Spectrum Radio Quasar (FSRQ) has been monitored by Fermi-LAT over a period of eight years (2008-2016). Due to the extreme nature of variability of the source, the light curve of PKS 1510-089 has shown many interesting results and has been studied by various authors $[1,9,11,13]$.

From the Fermi-LAT data collected over a period of eight years we have selected the high states/flares of PKS 1510-089 to compare their spectral and temporal properties. Although some of these high states of PKS 1510-089 have been studied before by other authors, a comprehensive study including all the high states observed by Fermi-LAT Collaboration till December, 2016 and a comparison of their spectral and temporal characteristics is not available in literature. Thus our work provides a detailed, complete and updated analysis of the flares of PKS 1510-089 detected by Fermi-LAT. The rest of the paper is organized as follows : in section 2, we describe the data analysis procedures, conduct a detailed study of various flares and construct the spectral energy distributions of the various states of the source. In section 3 we discuss the results and draw conclusions from our analysis. Throughout the paper we used the flux in units of $10^{-6} \mathrm{ph} / \mathrm{cm}^{2} / \mathrm{s}$ unless otherwise mentioned .

\section{Fermi-LAT Data Analysis of PKS 1510-089}

The Fermi-LAT is a pair conversion $\gamma$-ray telescope sensitive to photon energies greater than $20 \mathrm{MeV}$ with a field of view of about $2.4 \mathrm{sr}$ [4]. The primary observation mode of Fermi-LAT is survey mode in which the LAT (Large Area Telescope) scans the entire sky every 3 hours. PKS 1510-089 has been continuously monitored by Fermi-LAT since Aug 2008. We consider here the Fermi-LAT data for PKS 1510-089 from 05 Aug 2008-31 Dec 2016 (MJD 54683-57753). The data analysis has been done with the help of gtlike/pyLikelihood method, as implemented in the latest version (v10r0p5) of Fermi Science Tools software package.

The variability of the source can be clearly seen by producing light curves with different time-bins ( 7 days, 1 day, 12 hr, $6 \mathrm{hr}$ and $3 \mathrm{hr}$ ). In Fig.1, we show the weekly light curve which clearly reveals that the source is highly variable. In addition we performed the spectral analysis in the energy range 0.1-300 GeV over several periods of the flaring states by using the unbinned likelihood analysis.

The differential photon spectra have been fitted with three different functions whose forms are presented below.

- A power law (PL), defined as

$$
d N(E) / d E=N_{p}\left(E / E_{p}\right)^{-\Gamma}
$$

with $E_{p}=100 \mathrm{MeV}$ (contant for SEDs)

- A log-parabola (LP), defined as

$$
d N(E) / d E=N_{0}\left(E / E_{0}\right)^{-\alpha-\beta \ln \left(E / E_{0}\right)},
$$


with $E_{0}=300 \mathrm{MeV}$ (contant for SEDs),

- A power law with an exponential cut-off (PLEC), defined as

$$
d N(E) / d E=N_{0}\left(E / E_{p}\right)^{-\Gamma} \exp \left(-E / E_{c}\right),
$$

with $E_{p}=200 \mathrm{MeV}$ (contant for SEDs)

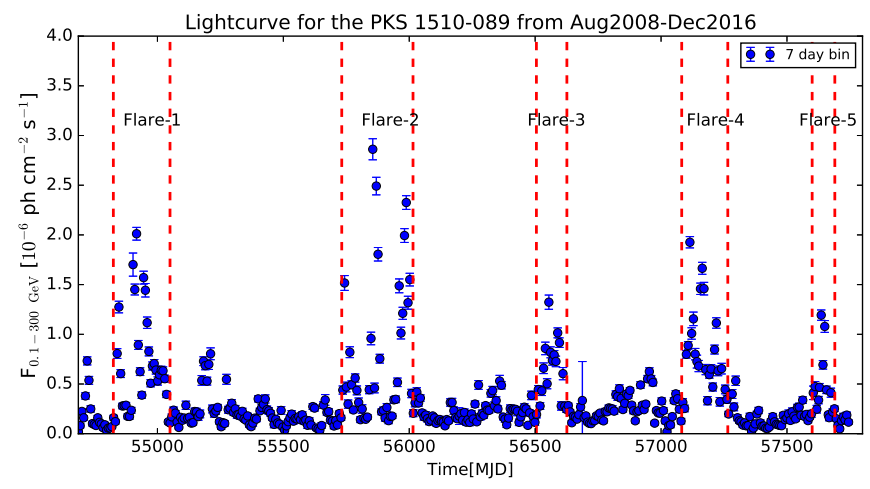

Figure 1: Light-curve history of PKS 1510-089

\subsection{Identifying the Flares of PKS 1510-089}

The light curve history of PKS 1510-089 shows that so far there have been five major flaring states (see Fig.1). We refer to these states in our work as Flare-1, Flare-2, Flare-3, Flare-4 and Flare-5 which happened during MJD 54825-55050, MJD 55732-56015, MJD 56505-56626, MJD 57082-57265 and MJD 57657-57753 respectively. We have zoomed in these major flares in bins of 1 day (not shown here) where sub-structures are clearly seen, $6 \mathrm{hr}$ (primarily for light curve study) and $3 \mathrm{hr}$ (for variability time scale study). The 6 hour binning clearly reveals that there are substructures and various phases (pre-flare, plateau, flare and post-flare) inside each individual flare shown in Fig.1. For further study, we concentrate on the plots with 6 hour bins. Two sub-structures have been observed during Flare-1 and Flare-2 shows five sub-structures. No sub-structure was seen during Flare- 3 and Flare-5 while three sub-structures were noticed during Flare-4. Here we are focussing on only one sub-structure of Flare-2 (i.e. flare-2C).

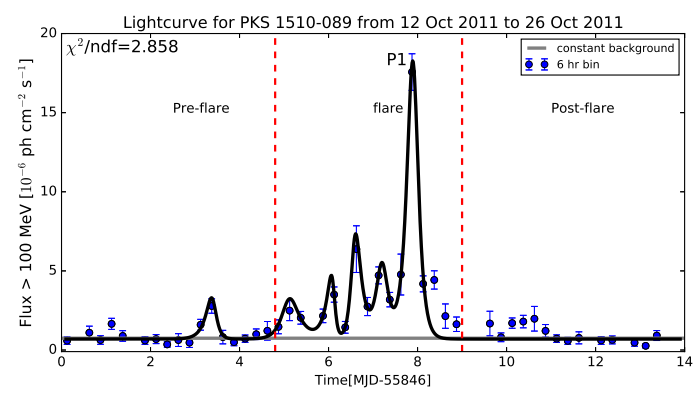

Figure 2: Temporal fitting of flare-2C 


\subsection{Light-curves of Flares}

We have studied the temporal evolution of each substructures separately. In order to show the temporal evolution, we have fitted the peaks by a sum of exponentials that give the decay and rising time for the different peaks shown in the figure 2.

The functional form of the sum of exponentials is

$$
F(t)=2 F_{0}\left[\exp \left(\frac{t_{0}-t}{T_{r}}\right)+\exp \left(\frac{t-t_{0}}{T_{d}}\right)\right]^{-1}
$$

[2], where $F_{0}$ is the flux at time $t_{0}$ representing the approximate flare amplitude, and $T_{r}$ and $T_{d}$ are the rise and decay time of the flare.

Rise and decay time for peak P1 of flare-2C, shown in figure 2, is mentioned in the table 1.

Table 1: Model parameters of temporal fitting for flare-2C

\begin{tabular}{ccccc}
\hline Peak & $\begin{array}{c}t_{0} \\
{[\mathrm{MJD}]}\end{array}$ & $\begin{array}{c}F_{0} \\
{\left[10^{-6} \mathrm{ph} \mathrm{cm}^{-2} \mathrm{~s}^{-1}\right]}\end{array}$ & $\begin{array}{c}T_{r} \\
{[\mathrm{hr}]}\end{array}$ & $\begin{array}{c}T_{d} \\
{[\mathrm{hr}]}\end{array}$ \\
\hline P1 & 55853.9 & $17.56 \pm 1.15$ & $2.92 \pm 0.89$ & $2.50 \pm 0.27$ \\
\hline
\end{tabular}

Temporal fitting as shown in figure 2 have been done for all the sub-structures. Their fitted parameters are plotted in figure 3 .
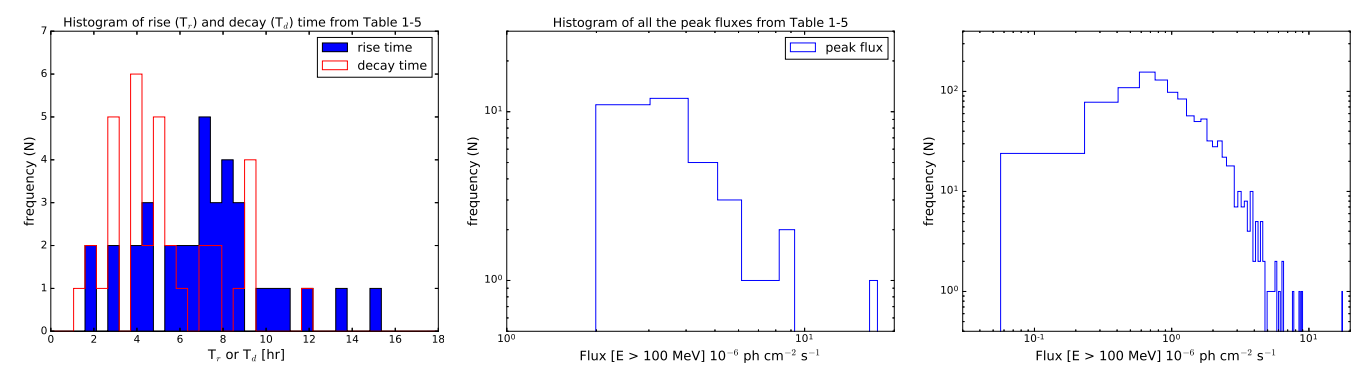

Figure 3: left: The frequency distribution of rise and decay time. middle: Frequency distribution of peak fluxes. right: Frequency distribution of all the flux data points from $6 \mathrm{hr}$ bin.

The rise and decay time for the different peaks of the flares are distributed around a mean of $6.04 \pm 0.22 \mathrm{hr}$ and $3.88 \pm 0.16 \mathrm{hr}$ with a standard deviation of $2.40 \mathrm{hr}$ and $2.20 \mathrm{hr}$ respectively. Which shows that most of the time flares have slow rise and fast decay (figure 3 (left)). The peak fluxes are distributed around a mean of $3.54 \pm 0.08$ with a standard deviation of 1.69 . Which represent that when the source is flaring its flux most of the time is around $3.54 \times 10^{-6} \mathrm{ph} \mathrm{cm}^{-2} \mathrm{~s}^{-1}$ (figure 3 (middle)). The right part of figure 3 shows the peak distribution with slow rising part upto the peak and the fast decaying part beyond the peak. The peak value signifies the flux where source spend it most of the time. Above the peak, the flux value falls rapidly along with few outliers which can be associated with large flux variations in the source.

\section{$2.3 \gamma$-ray SEDs of flares}

We have produced the SEDs PKS 1510-089 during different phases of the flares. The SEDs data points are fitted with three different models PL, LP, PLEC and their functional form are given 
Table 2: fitted parameters of flare-2C

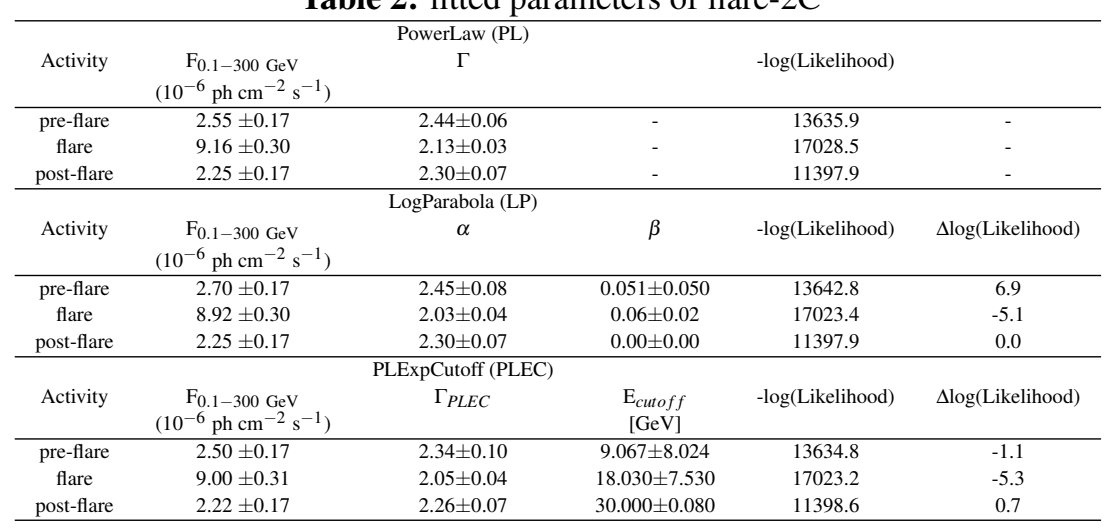

in equation 2.1, 2.2 and 2.3 respectively. The SEDs plot for all the phases of flare-2C is shown in figure 4 and the corresponding fitted model parameters are presented in table 2. Hardening in the spectrum is noticed during flare- $2 \mathrm{C}$ as the flux increases from pre-flare to flare phase with the value of spectral index $\Gamma$ changing from $2.44 \pm 0.06$ to $2.13 \pm 0.03$ when fitted with powerlaw distribution. In all the other sub-structures we have also seen a progressive spectral hardening when source is moving from pre-flare to its flaring state (plots and table are not shown here because of page constrain).
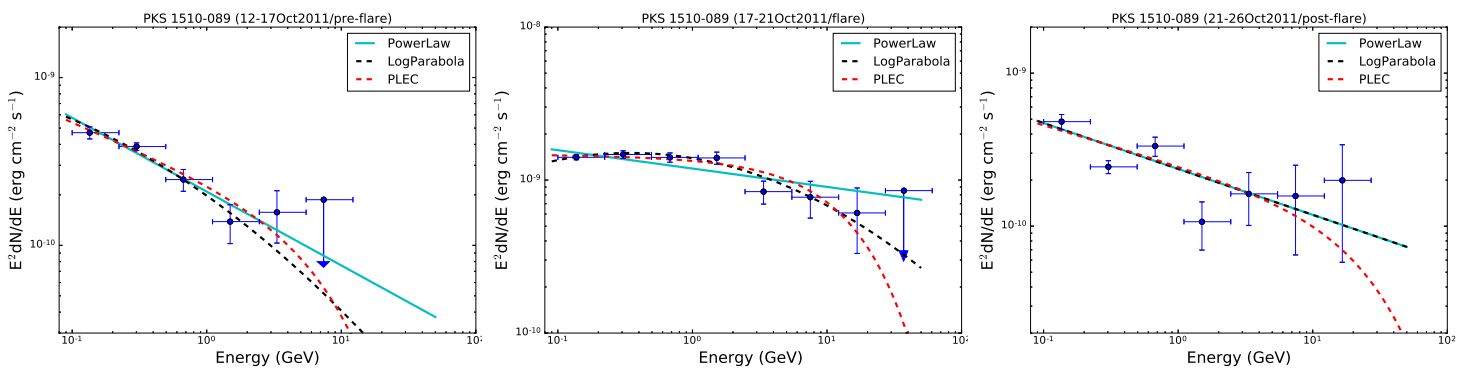

Figure 4: From left to right SEDs are plotted for pre-flare, flare and postflare of flare-2C respectively.

\section{Results and Discussions}

Being one of the most variable blazars in the Third Source Fermi Catalog (3FGL) the light curve of PKS 1510-089 comprises of five major flares and each flare comprises of several subflares. Almost all the sub-flares shows various phases (pre-flare, flare, plateau, post-flare) and the flaring phases consist of peaks of different heights. Decay and rising times have been calculated for the major and clear peaks (P1, P2, P3..etc). Most of the peaks have rising and decay times of few hours (less than a day). The SEDs of all the phases are fitted with PL, LP and PLEC functions; the reduced-chisquare and the cutoff of energy are mentioned in table 3. The brightest flare was observed during Oct 2011 at MJD 55853.813. For $3 \mathrm{hr}$ binning the flux was $\mathrm{F}_{\mathrm{GeV}}=25.50 \pm 2.34$ with TS $=1340$. A new flare was found in Aug 2015 (Fig.12) which has a peak P1 at MJD 57244.56 with a flux $\mathrm{F}_{\mathrm{GeV}}=8.92 \pm 1.25(\mathrm{TS}=397.18)$. More recently a flare was also observed during 28 Aug-15 Sep, 2016 with a flux of $\mathrm{F}_{G e V}=3.15 \pm 0.47$ at MJD 57634.61. 
Our results show in detail the presence of sub-flares within the flares, which we have scanned separately by using the following function,

$$
F\left(t_{2}\right)=F\left(t_{1}\right) \cdot 2^{\left(t_{2}-t_{1}\right) / t_{d}},
$$

to calculate the minimum time of doubling/halving of flux between the time instants $t_{1}$ and $t_{2}, F\left(t_{1}\right)$ and $F\left(t_{2}\right)$ are respectively the fluxes measured at $t_{1}$ and $t_{2}$ and $t_{d}$ represents the doubling/halving time scale.

We have found the fastest variability time is $1.30 \pm 0.18$ during the flare- $2 \mathrm{C}$. The variability time gives an idea about the size of the emission if we know the Doppler factor $\delta$ for the source. The variability time $\mathrm{t}_{v a r}$, the size of the emission region $\mathrm{R}$, and the Doppler factor $\delta$ are related by

$$
R \leq c t_{v a r} \delta(1+z)^{-1}
$$

where $z$ is the redshift of the source. We found an emission region of radius $\mathrm{R} \sim 4.85 \times 10^{15} \mathrm{~cm}$.

Table 3: The reduced- $\chi^{2}$ for SEDs fitted by PL, LP and PLEC for the flaring episodes are displayed below. In most cases LP and in a few cases PLEC provide the best fit to the data. Cutoff energies found with PLEC

\begin{tabular}{|c|c|c|c|c|}
\hline Activity & \multicolumn{3}{|c|}{ Reduced- $\chi^{2}$} & \multirow{2}{*}{$E_{\text {cutoff } f}$ for PLEC $(\mathrm{GeV})$} \\
\hline flare-1(A) & $\begin{array}{l}\mathrm{PL} \\
\end{array}$ & LP & $\begin{array}{l}\text { PLEC } \\
\end{array}$ & \\
\hline flare(I) & 2.28 & 2.31 & 1.98 & $30.00 \pm 0.25$ \\
\hline flare(II) & 2.90 & 0.12 & 1.09 & $15.98 \pm 6.36$ \\
\hline \multicolumn{5}{|l|}{ flare-1(B) } \\
\hline flare & 5.06 & 0.58 & 1.03 & $5.74 \pm 1.83$ \\
\hline \multicolumn{5}{|l|}{ flare-2(A) } \\
\hline flare(I) & 3.66 & 1.91 & 2.40 & $11.27 \pm 8.13$ \\
\hline flare(II) & 2.84 & 0.92 & 0.48 & $2.70 \pm 0.73$ \\
\hline \multicolumn{5}{|l|}{ flare-2(B) } \\
\hline flare & 2.15 & 0.23 & 0.43 & $5.82 \pm 2.36$ \\
\hline \multicolumn{5}{|l|}{ flare-2(C) } \\
\hline flare & 1.73 & 0.41 & 0.83 & $18.03 \pm 7.53$ \\
\hline \multicolumn{5}{|l|}{ flare-2(D) } \\
\hline flare & 8.14 & 0.43 & 2.83 & $12.31 \pm 3.51$ \\
\hline \multicolumn{5}{|l|}{ flare-2(E) } \\
\hline flare(I) & 10.23 & 1.63 & 2.41 & $7.61 \pm 1.53$ \\
\hline flare(II) & 0.43 & 0.15 & 0.06 & $9.71 \pm 5.12$ \\
\hline \multicolumn{5}{|l|}{ Flare-3 } \\
\hline flare(I) & 2.73 & 0.91 & 1.19 & $5.02 \pm 0.18$ \\
\hline flare(II) & 0.41 & 0.42 & 0.34 & $9.04 \pm 0.22$ \\
\hline \multicolumn{5}{|l|}{ flare-4(A) } \\
\hline flare & 11.93 & 3.25 & 5.82 & $12.78 \pm 8.11$ \\
\hline \multicolumn{5}{|l|}{ flare-4(B) } \\
\hline flare(I) & 2.41 & 2.95 & 1.78 & $9.07 \pm 0.31$ \\
\hline flare(II) & 8.60 & 0.50 & 3.30 & $9.74 \pm 3.16$ \\
\hline \multicolumn{5}{|l|}{ flare-4(C) } \\
\hline flare & 4.41 & 1.00 & 1.84 & $29.71 \pm 8.16$ \\
\hline \multicolumn{5}{|l|}{ Flare-5 } \\
\hline flare & 1.55 & 0.43 & 0.50 & $5.01 \pm 2.00$ \\
\hline
\end{tabular}
vary from one flare to another, which could be due to different emission regions of these flares

We compared the reduced $\chi^{2}$ values for PL, LP and PLEC fits and the spectral cut-off energies in table 3 for different flares. In most of the cases, the best fit is found to be LP during flaring episodes. We also note that in the case of PLEC fit, the spectral cut-off energy varies from one flare to another. It is interesting to note that in a few cases where the reduced chi-square values for PLEC are comparable to the values obtained from LP fits, the cut-off energy is well constrained. This has strong physics implications regarding the location of the emission region. If the emission region is close to the core of the source, pair production optical depth would prevent the escape of very high energy gamma rays. As a result the highest energy gamma rays are expected from zones outside 
the BLR region, in the optically thin outer jet region (see [3]). The variations in spectral fittings and spectral cut-off energies of the flares indicate that different flares might have originated from different zones along the length of the jet of PKS 1510-089. Earlier studies on blazar flares also indicated the possibility of multiple zones of emission during flares [5,6,7]. Detailed broadband spectral modeling with photon data ranging from radio to $\mathrm{TeV}$ energy would be more useful in exploring the complex nature of flares of this highly variable source.

\section{Concluding Remarks}

We have studied the long term light curve of PKS 1510-089 with the data collected by FemiLAT between Aug 2008 to Dec 2016. The data have been binned in 7 days, 1 day and $6 \mathrm{hr}$ to explore various features of the light curve. Five major flares along with many substructures have been detected in the weekly binning of the data which have been further studied in detail. From a detailed study on variability, the shortest variability time has been found to be close to 1 hour. This puts a strong constraint on the size of the emission region which has been estimated to be $\sim 10^{15} \mathrm{~cm}$ for reasonable values of the Doppler factor. The spectral energy distributions have been fitted with three different functional forms PL, LP and PLEC. We find that in majority of the flares LP gives the best fit and in some cases PLEC can reasonably describe the data. Moreover, when PLEC gives the best fit the cut-off energies are found to vary from one flare to another. Our results indicate that the emission regions vary from one flare to another which is consistent with earlier results.

\section{References}

[1] Abdo, A. A., Ackermann, M., Agudo, I., et al., 2010, ApJ, 721, 1425

[2] Abdo, A. A., Ackermann, M., Ajello, M., et al., 2010, ApJ, 722, 520

[3] Aleksić, J., Ansoldi, S., Antonelli, L. A., et al., 2014, A\&A, 569, A46

[4] Atwood, W. B., Abdo, A. A., Ackermann, M., et al., 2009, ApJ, 697, 1071

[5] Brown, A. M., 2013, MNRAS, 431, 824

[6] Dotson, A., Georganopoulos, M., Kazanas, D., \& Perlman, E. S., 2012, ApJL, 758, L15

[7] Dotson, A., Georganopoulos, M., Meyer, E. T., \& McCann, K., 2015, ApJ, 809, 164

[8] E. M. Burbidge,T. D Kinman, Burbidge et al.(1966), ApJ, 145, 654

[9] Foschini, L., Bonnoli, G., Ghisellini, G., et al., 2013, A\&A, 555, A138

[10] H.E.S.S. Collaboration, Abramowski, A., Acero, F., et al., 2013, A\&A, 554, A107

[11] MAGIC Collaboration, Ahnen, M. L., Ansoldi, S., et al., 2016, arXiv:1610.09416

[12] D. J. Thompson, S. Djorgovski,R. de Carvalho, (1990), PASP 1021235

[13] Zacharias, M., Böttcher, M., Chakraborty, N., et al., 2016, arXiv:1611.02098 